\title{
Thyroid Emphysema Following Penetrating Neck Trauma
}

\author{
Demet Karadağ ${ }^{1}$, Egemen Doner ${ }^{2}$, Baki Adapınar ${ }^{1}$ \\ ${ }^{1}$ Department of Radiology, Faculty of Medicine, Eskişehir Osmangazi University, Eskişehir, Turkey \\ ${ }^{2}$ Department of Thoracic Surgery, Faculty of Medicine, Eskişehir Osmangazi University, Eskişehir, Turkey
}

\begin{abstract}
Although traumatic thyroid gland rupture or hemorrhage is usually seen in goitrous glands, injuries of the normal thyroid gland after neck trauma have rarely been described in the literature. We describe a 44-year-old man who presented with thyroid emphysema and subcutaneous emphysema (SCE) that occurred after penetrating neck trauma. CT images showed complete resolution of thyroid emphysema and subcutaneous emphysema at follow-up examination. Neck injuries can be life threatening. After penetrating neck traumas, physicians should consider subtle esophageal or tracheal laceration. Thyroid emphysema can occur as the result of penetrating neck trauma. The mechanism of emphysema of the thyroid parenchyma can be explained by the thyroid gland's presence in a single visceral compartment that encompasses the larynx, trachea and thyroid gland. We describe an unusual case of thyroid emphysema of a normal thyroid gland following a penetrating neck injury.
\end{abstract}

Key Words: Thyroid, emphysema, subcutaneous emphysema, penetrating neck trauma, CT

Received: 26.11.2008 Accepted: 05.02.2009

\section{Introduction}

The mechanism of neck traumas can be blunt or penetrating. Penetrating injuries to the neck involving the thyroid gland are relatively uncommon. Although traumatic rupture or hemorrhages of the thyroid gland are very rare, most of the reported cases are related to a pre-existing goiter (1). Because of a fragile structure caused by enlargement and increased vascularity in the goitrous tissue, trauma can easily lead to rupture and hemorrhage. Nevertheless, a few cases of normal thyroid gland injuries have also been described. We describe an unusual case of thyroid emphysema of a normal thyroid gland following a penetrating neck injury.

\section{Case Report}

A 44-year-old construction worker presented with a penetrating injury with an iron rod which had stabbed into the right side of his neck. On physical examination, there was a wound on the right side of the neck. Crepitation and swelling were observed on both sides of the neck. The patient's general physical condition was normal. There was no history of difficulty in breathing. Thyroid function tests were normal. Neck and chest computed tomography (CT scans) revealed marked air accumulation in the subcutaneous tissues of the neck, thyroid parenchyma, and the mediastinum. CT showed no obvious evidence of thyroid parenchyma injury or airway laceration. Thyroid gland contours were smooth (Fig. 1). Clinically, there was no finding of tracheal or esophageal laceration. The patient refused any surgical approach at that time and the patient had a conservative follow-up without surgical exploration. The neck swelling resolved with conservative treatment. The patient was discharged in good condition 5 days after the trauma. Pneumomediastinum, subcutaneous emphysema (SCE) and thyroid emphysema disappeared during 2 weeks of follow up (Fig. 2). A gastrograffin swallow was performed and there was no evidence of perforation or laceration of the esophagus. Thyroid function tests were repeated and the tests were normal. Surgical exploration was proposed for removing the metallic object to the patient.

\section{Discussion}

Neck trauma can be caused by a penetrating or blunt injury. Because the neck contains vital vascular, neural, respiratory and digestive structures, neck injuries can be life threatening. The neck can be partitioned into three zones for the optimal management of penetrating neck injuries. Zone I extends from the clavicle to the cricoid cartilage, zone II between the cricoid cartilage and the angle of the mandible, and zone III is from the level of the angle of the mandible to the base of the skull. Zone II is the most commonly involved region and surgical approach to this region is more accessible than the other zones.

The term 'emphysema' is used to describe an accumulation of air or gas in the tissue spaces. Subcutaneous emphysema of the neck can occur due to thoracic surgical procedures, infections, pneumomediastinum, or trauma to the pharynx, esophagus, or trachea. Rarely it can follow oral and nasal surgery or maxillofacial trauma. The most common cause of SCE 

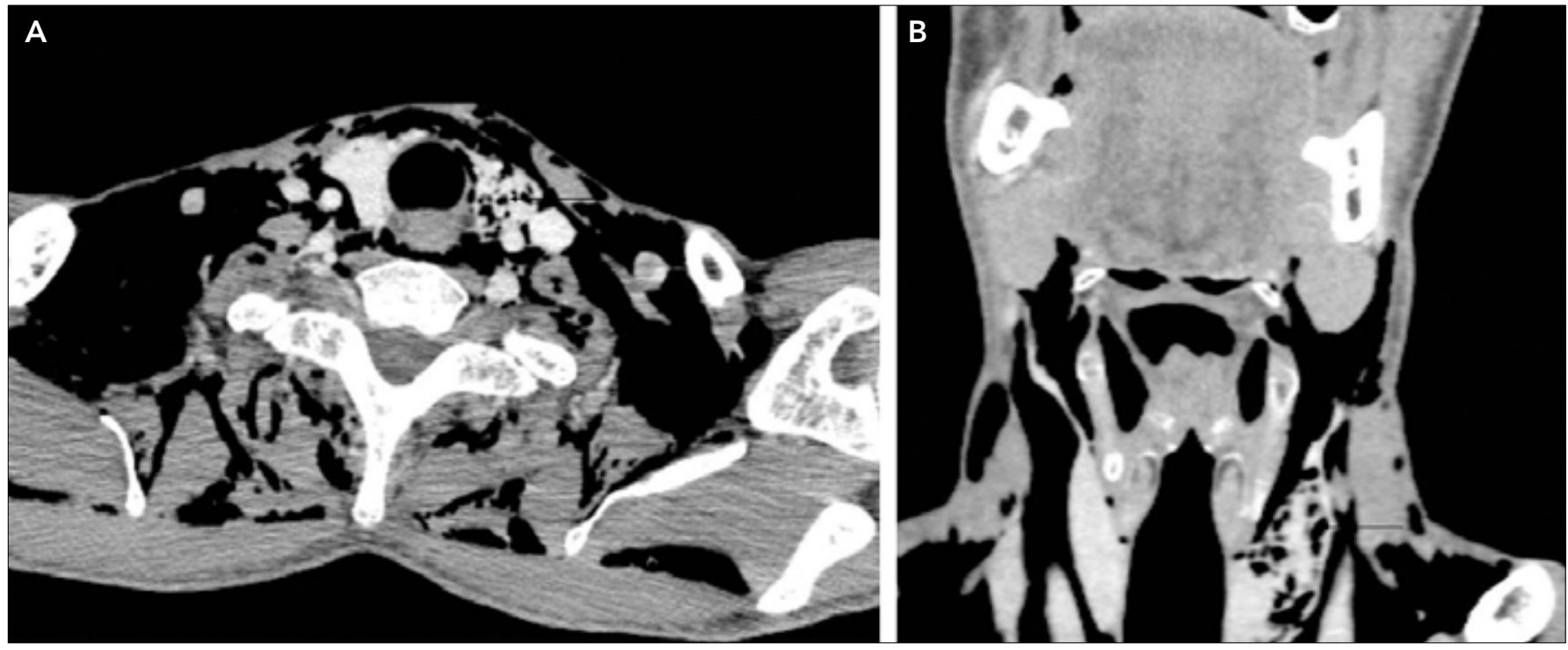

Figure 1. Axial (A), Coronal (B) Computed Tomography Scan of the Neck Shows Thyroid Emphysema with SCE
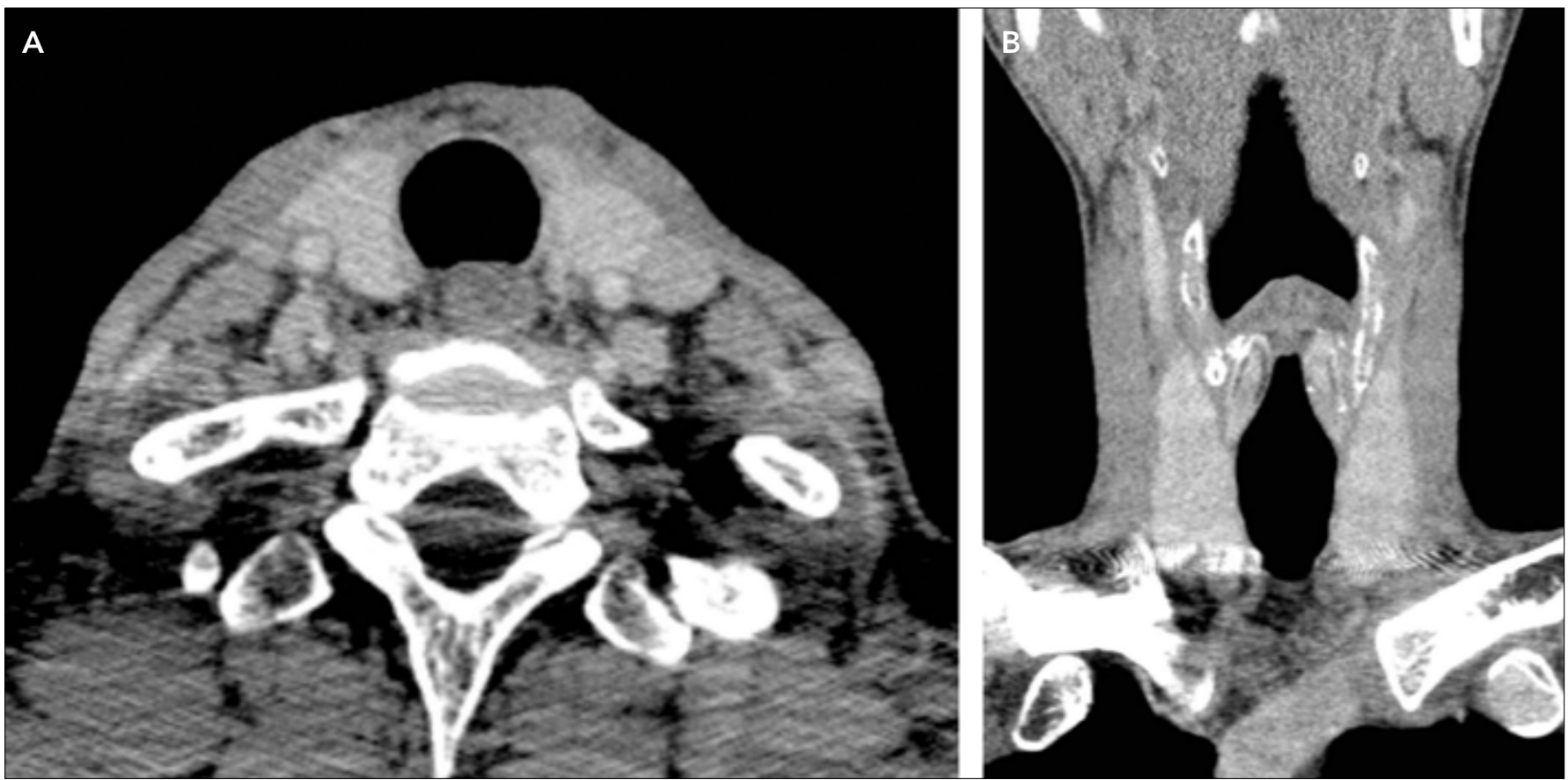

Figure 2. Axial (A), Coronal (B) computed Tomography Scan Shows Disappearance of Air Collections in the Thyroid Gland and Subcutaneous Tissue

is trauma. When SCE occurs, then the air travels easily through the soft tissue planes of the neck.

The thyroid is the largest gland in the neck. Nevertheless, traumatic thyroid injuries are rare and only a few cases have been reported. In most previously described cases, traumatic rupture or hemorrhage of the thyroid gland occurred in the presence of pre-existing goiter (3). Because of the fragile structure of the goitrous thyroid gland, trauma easily leads to rupture and hemorrhage in the thyroid gland. On the other hand, traumatic rupture of the normal thyroid gland is an extremely rare event (2-4).
In our case, there was no radiological evidence of injury to the thyroid gland such as rupture and hemorrhage, or infection of the thyroid gland. Although there have been a few reports of acute emphysematous thyroiditis (5), it is interesting that our literature search we did not yield any report of thyroid emphysema following trauma. Acute emphysematous thyroiditis is characterized by severe pain in the neck, fever and marked leucocytosis. Furthermore, hypothyroidism can result from pyogenic destruction of thyroid tissue (5). However, in our case, there were no clinical or laboratory findings related to traumatic thyroid emphysema. 
We believe that the evidence of subcutaneous and mediastinal emphysema leads to the consideration of a subtle esophageal or tracheal laceration. The mechanism of the spread of air into the thyroid parenchyma can be explained by the presence of the thyroid gland in a single visceral compartment that is continuous with the superior mediastinum. This middle layer of the cervical fascia encompasses the larynx, trachea, thyroid gland, parathyroid glands and inferior thyroid artery.

To our knowledge, no other report of thyroid emphysema following a penetrating neck injury has been mentioned in medical literature. Neck swelling due to SCE after penetrating neck trauma must be differentiated from an expanding hematoma in order to safely conclude that a surgical exploration is not necessary.

\section{Conflict of Interest}

No conflict of interest was declared by the authors.

\section{References}

1. Watson AP. Traumatic rupture of the thyroid gland. Australas Radiol. 1999;43:363-4.

2. Thompson EC, Porter JM, Fernandez LG. Penetrating neck trauma: an overview of management. J Oral Maxillofac Surg 2002;60:918-23. [CrossRef]

3. Park CH, Oh KK, Kim EK, Kim MJ, Jeong J, Son EJ. Thyroid gland rupture after blunt cervical trauma. J Ultrasound Med 2006;25:943-6.

4. Rupprecht $H$, Rumenapf G, Braig H, Flesch R. Acute bleeding caused by rupture of the thyroid gland following blunt neck trauma: case report. J Trauma 1994;36:408-9. [CrossRef]

5. Gigot JF, Mannell A. Acute emphysematous thyroiditis. Br J Surg. 1983;70:256-8. [CrossRef] 